\title{
De la compétence rare aux métiers atypiques: le journalisme peut-il écrire les relations publiques ?
}

\author{
Ivan Ivanov, Professeur adjoint, \\ Université d'Ottawa - GRICO \\ Département de communication \\ iivanov@uottawa.ca
}




\title{
Résumé
}

Cet article s'intéresse à l'écriture en organisation en tant que compétence chère, mais rare des relations publiques (RP). L'écriture est située au premier rang des activités techniques du relationniste aux côtés des tâches managériales, même si souvent elle est perçue comme ingrate et désagréable, car liée aux activités prescriptives et imposées. Pourtant, l'incapacité d'écrire pour divers publics internes et externes a pour conséquence la mauvaise presse des RP. Face au manque de programmes universitaires et de formations continues, les relationnistes apprennent souvent sur le terrain à écrire et à éditer des supports d'information et de communication. Et si les organisations ouvraient leurs portes à ceux qui maîtrisent l'art d'écrire? L'écriture est au centre des compétences journalistiques et les directions des RP embauchent depuis des décennies d'anciens journalistes professionnels. Cependant, les activités hybrides qui naissent de cette union sont très critiquables et contestées et donnent vie à des métiers qui n'ont que peu de reconnaissance et de légitimité interne et externe, mais qui existent dans les organisations comme pratiques d'origine journalistique intégrées à l'exercice des RP.

Mots-clés : compétence, hybride, journalisme, relations publiques, écrire.

\begin{abstract}
This article focuses on corporate writing as an important-yet somehow rare-skill for public relations (PR). Writing is one of the key competencies at the heart of the communicator's technical activities alongside other managerial tasks, even though it is often perceived as unpleasant and fruitless since it is associated with prescriptive and imposed activities. The inability to write for a variety of internal and external audiences, however, contributes much to the bad reputation of PR. Faced with the lack of university programs and continuing education, PR Staff often learn to write and publish information and communication materials on the go. What if more organizations opened their doors to those who master the art of writing? Writing is central to performing journalistic skills; PR editorial staff have hired professional journalists for decades. In that regard, the hybrid activities that come out of this coupling are highly questionable and contentious: they give rise to professions that have no legitimacy and recognition, but that nonetheless exist in organizations as journalistic practices embedded in standard PR.
\end{abstract}

Keywords: Competency, Hybridity, Journalism, Public Relations, Writing. 


\section{Introduction}

Si la cohabitation du journalisme et des RP est un fait historique bien étudié (Sallot et Johnson, 2006), leur croisement est une source d'actualité et de controverses méconnue et peu explorée (Macnamara, 2015). La conjugaison des pratiques journalistiques et communicationnelles est difficilement concevable, parce qu'elles puisent leur origine dans des paradigmes bien distincts et souvent opposés (Mathien, 1998), même si leur hybridation a le mérite de placer le système normatif dans la logique chronologique des pratiques professionnelles (Charron et De Bonville, 1997). Or, c'est bien dans la pratique quotidienne de ces deux activités que des formes hybrides — du côté du journalisme aussi bien que des RP — naissent comme des formes extraconjugales d'une union secrète devenue truisme. Il revient ainsi à questionner certaines compétences communes aux journalistes et aux relationnistes qui caractérisent leurs activités professionnelles et qui font naître, sur le terrain des organisations, de nouveaux métiers hybrides basés sur des pratiques professionnelles divergentes, voire antagoniques, qui se rencontrent, croisent et cherchent leur reconnaissance et légitimité. Quelles sont alors les compétences au carrefour du journalisme et des RP qui légitiment la pratique de métiers hybrides qui existent, mais qui sont toujours contestés et contestables dans les organisations?

Pour répondre à cette question, nous proposons d'explorer les compétences des relationnistes qui découlent directement du journalisme professionnel : l'écriture et l'édition de supports d'information et de communication (Ross, 2005 ; Agnès, 2002). Nous nous intéressons plus particulièrement à l'écriture, une des compétences chères des RP qui sont conçues tantôt comme un métier intégré à la coalition dominante (Grunig, 1992), tantôt comme une compétence technique et pratique (Cernicova, Dragomir et Palea, 2011 ; Broom et Dozier, 1986). Si le premier volet a été largement étudié, le deuxième reste quelque peu dévalorisé et marginal du fait qu'il implique les relationnistes dans des activités «bas de gamme» (McGoon, 1995). Pourtant, c'est souvent le manque de compétences techniques qui nuit considérablement à l'image des RP et nourrit la perception désastreuse des relationnistes aux yeux des journalistes (Wilson et Supa, 2013). Fragmentairement pris en charge par les enseignements universitaires et les formations continues, difficilement maîtrisable à travers l'unique pratique sur le terrain (Kim et Johnson, 2011), l'art d'écrire pour divers publics internes et externes reste l'apanage du journalisme professionnel.

C'est dans ce contexte précis que nous proposons d'ouvrir la boîte noire du journalisme d'entreprise (Ivanov, 2016a) et de questionner le travail des anciens journalistes professionnels devenus communicants : une inflexion risquée, mais présente sur le terrain depuis plusieurs années, qui est, certes, difficile à concevoir et à accepter - y compris par ceux qui le pratiquent - , mais qui mérite une clarification plus précise et une définition plus tangible. Nous ne souhaitons pas militer pour une telle hybridation ni défendre l'apparition d'activités comme le journalisme d'entreprise. 
Mais les évolutions contemporaines des pratiques des RP dans les organisations ainsi que celles touchant le journalisme - nécessitent de questionner, mettre en exergue et expliquer les nouvelles formes professionnelles qui existent en dépit des fractions paradigmatiques.

Pour ce faire, dans la première partie de cet article, nous procédons à une revue des travaux en RP qui clarifie le rôle et les compétences des relationnistes en tant que gestionnaires et techniciens. Nous nous attachons ensuite à démontrer que les compétences écrites en entreprise et les formations universitaires des deux côtés de l'Atlantique ne les prennent pas systématiquement ni suffisamment en considération. Afin d'illustrer nos propos, nous prenons pour exemple le métier atypique de journaliste d'entreprise, difficile à cerner, mais clairement identifié par certains organismes étatiques d'accompagnement à la recherche d'emploi et par plusieurs associations professionnelles. Nous discutons finalement les prérequis et les dispositions qui pourraient contribuer à la reconnaissance et à la légitimation de tels métiers hybrides, qui certes ne portent pas partout le même nom, mais qui reposent sur des pratiques professionnelles similaires.

\section{Le travail du relationniste dans les organisations}

Les études portant sur les compétences et le savoir-faire des relationnistes sont minoritaires dans les domaines des RP (Edwards, 2011). Cette carence - ou plutôt ce manque d'intérêt pour de telles études - fait émerger clairement deux phénomènes durablement imbriqués : la difficulté de se mettre d'accord sur le rôle des relationnistes dans les organisations (encore faut-il définir l'organisation!) et la complexité de circonscrire leurs tâches en lien avec des compétences professionnelles clairement explicitées et délimitées.

En adoptant une posture analytique et normative (Fondin, 2001), notre objectif est de présenter un cadre de lecture explicite des phénomènes observés par nous-mêmes et par d'autres chercheurs afin de dresser une image non exhaustive de certaines compétences et missions des relationnistes dans les organisations.

\section{1. Écrire : un travail « down and dirty »}

Dans le monde anglo-saxon, les travaux de Broom et Smith $(1978,1979)$ sur les quatre rôles fondamentaux du relationniste - expert, technicien, animateur et facilitateur de problèmes ${ }^{1}$ - ont été un des premiers efforts systématiques pour mieux comprendre le travail des professionnels des RP dans les organisations. Dans ce modèle, discuté ensuite par d'autres chercheurs (Grunig, 2006; Bronn, 2001;

\footnotetext{
${ }^{1}$ Toutes les traductions de l'anglais vers le français sont faites par l'auteur.
} 
Berkowitz et Hristodoulakis, 1999), les rôles attribués sont liés par la transversalité et la nature technique ou stratégique des tâches allouées.

Le relationniste expert agit avec une certaine autorité reconnue dans deux domaines conjoints : l'analyse de problèmes liés aux pratiques et aux stratégies de relations publiques ainsi qu'au niveau de leurs solutions (développement de plans, de programmes et d'actions appropriées). Le technicien de communication (Bowen, Rawlins et Martin, 2010) intervient lorsque l'organisation nécessite la réalisation d'outils et de supports destinés à épauler les solutions proposées grâce à des compétences spécifiques et typiques du journalisme : écrire, rédiger, synthétiser, éditer des supports de communication internes et externes lisibles et de bonne qualité par le biais d'un savoir-faire particulier (Broom, 1982). Afin d'assurer la transmission et la fluidité des informations et des supports fabriqués, ainsi que le flux de communication bidirectionnelle (Grunig et Grunig, 2000), le relationniste-animateur intervient à son tour comme médiateur entre l'organisation qui l'emploie et ses différents publics internes et externes. Toutes ces actions s'inscrivent dans un cadre qui est celui de la stratégie officielle de l'organisation. D'où l'intérêt du travail du facilitateur de problèmes (Broom et Dozier, 1986), qui fait partie de la coalition dominante afin d'accompagner les gestionnaires de haut niveau lors de la prise de décisions stratégiques (Grunig, 1992) et de la recherche de solutions de problèmes de haute importance et qui influence directement le travail de l'expert, du technicien et de l'animateur.

Ce modèle a connu plusieurs développements, comme par exemple ceux apportés par Dozier (1981) lorsqu'il propose d'y inclure le spécialiste de relations médias - salarié non-cadre chargé des relations avec les médias externes - et le communicant de liaison qui fournit du conseil au management, mais qui n'est pas un gestionnaire. Au-delà des différentes variations de ce modèle, deux grandes figures du relationniste relativement stables dans le temps se sont dégagées — le gestionnaire et le technicien - et tous les autres rôles peuvent y être classés, peu ou prou, comme des sous-catégories (Piekos et Einsiedel, 1990). La majorité des travaux qui a suivi ce modèle s'est concentrée sur l'étude de la figure du relationniste en tant que gestionnaire ou celle du professionnel au service du management (Gregory, 2008 ; Bronn, 2001 ; Grunig et Grunig, 2000), alors que celle du technicien reste quelque peu marginale. Et il y a probablement de bonnes raisons à cela. Le travail du technicien est souvent considéré comme "down and dirty» (McGoon, 1993) et sa profession comme un «semi-métier» (Dozier, 1992), car il n'est pas directement lié à la gestion stratégique de l'organisation, mais est basé sur l'exécution de tâches techniques dépourvues de toute latitude décisionnelle. Dans les organisations, la hiérarchisation normative qui en découle va de pair avec un manque de valorisation institutionnelle du travail du technicien (et entrâne d'autres types d'inégalités, comme celles liées au genre et à l'accès limité des femmes aux postes de gestion - cf. Niquette, 2000), d'où l'appel soutenu de Grunig (2006) à revaloriser le travail du 
relationniste et à l'inclure obligatoirement dans la coalition dominante pour qu'il puisse influencer la prise de décisions stratégiques.

Pourtant, cette proposition est souvent critiquée (Cheney et Christensen, 2001). Peu d'études et d'exemples pratiques démontrent la volonté — et surtout la nécessité d'amalgamer le travail du relationniste et du gestionnaire, d'autant plus que dans la pratique observée (Ivanov, 2013), le relationniste reste derrière les portes des salles de réunion de la direction et n'est pas présent lorsque le management discute les décisions stratégiques (Maisonneuve, 2010 ; Kim et Johnson, 2011). Or, le relationniste est, au contraire, beaucoup plus sollicité comme un technicien de communication avec un savoir-faire et des compétences spécifiques, capable de mettre l'organisation «sous presse». McGoon (1993) souligne déjà en son temps que la majorité de relationnistes en Amérique du Nord ne souhaite pas travailler comme gestionnaire ou comme directeur, car ils se considèrent beaucoup plus heureux et épanouis lorsqu'ils écrivent et éditent des publications et des supports de communication divers. Ce sentiment semble être aussi partagé aujourd'hui par les employeurs qui ont de fortes attentes en termes de compétences écrites et rédactionnelles lorsqu'ils embauchent des professionnels des relations publiques (Flynn, 2014). Les capacités relationnelles et l'esprit créatif recherchés par les recruteurs sont systématiquement conjugués avec la compétence d'écrire pour un public large et diversifié. Cette compétence est, en conséquence, chère aux relationnistes aussi bien qu'aux employeurs.

\subsection{Rôles, compétence et tâches du professionnel des RP en interne et en externe}

La compétence est définie ici comme un concept plus large que le savoir-faire, car il inclut des habiletés propres au métier professionnel exercé, permettant au relationniste d'atteindre des objectifs spécifiques, ainsi que des habiletés génériques (Jeffery et Brunton, 2010) qui dominent toute autre performance professionnelle et qui sont typiquement liées à la communication écrite et orale. Cernicova, Dragomir et Palea (2011) réalisent une étude auprès de 70 relationnistes de l'Europe de l'Est qui répondent à 37 questions réunies dans deux questionnaires. Les résultats obtenus montrent que $85 \%$ des répondants classent les techniques d'écriture au premier rang des compétences des relationnistes, devant l'utilisation des technologies et des plateformes numériques. Gregory (2008) arrive à un résultat semblable en Angleterre lorsqu'elle réalise 17 interviews avec des relationnistes leaders dans le domaine public et privé et classe les compétences communicationnelles - écrire d'une manière claire et convaincante en interne comme en externe - à la première place. Ces différentes études démontrent que les compétences écrites et éditoriales sont représentées de manière quasi transversale et universelle dans les activités des RP dans les différents types d'organisations. Elles sont au service du management aussi bien que des autres branches et secteurs (par exemple, les communiqués de presse rédigés sont transmis 
par différents secteurs comme la comptabilité, la logistique ou encore les ressources humaines).

Si l'écriture en RP externes est traditionnellement davantage valorisée par le biais des relations médias, de la communication institutionnelle ou encore du web 2.0 et des réseaux socionumériques, les RP internes ${ }^{2}$ restent «l'enfant pauvre» de la communication d'entreprise. Yeomans (2006) souligne que très peu d'études en RP s'intéressent à l'élaboration de supports et de contenus à destination des salariés noncadres, mais sont plutôt concentrées sur la mise en place d'outils réservés au management pour mieux gérer les individus et les équipes au travail. La question de l'importance des compétences et des habiletés des relationnistes, nécessaires pour créer et développer des supports de communication interne lisibles lorsqu'ils communiquent avec et pour les salariés, reste en grande partie occultée. Il en est de même en France où « la communication et les relations publiques internes n'avaient absolument aucune place dans l'entreprise » (Carayol, Chaudet et Frame, 2014, p. 196). D'autre part, très peu d'intérêt est porté à la professionnalisation des communicants internes (De La Broise et Brulois, 2010) et sur l'institutionnalisation de leurs compétences écrites et éditoriales par-delà des modes d'écritures normés ou prescrits (pour qu'elles soient fonctionnelles).

Sur le plan technique, le professionnel de RP internes s'occupe de concevoir, développer, élaborer, mettre en place, maintenir et actualiser des supports écrits, électroniques et audiovisuels, destinés à nourrir la communication avec les différents publics internes d'une organisation. Plusieurs études classent les habiletés liées à l'écriture et à l'édition de supports écrits parmi les compétences les plus importantes en RP internes (Verčič, Verčič et Sriramesh, 2012). Les différentes étapes de l'étude Delphi, qui fait partie du projet European Public Relations Body of Knowledge (Verčič, 2000), positionnent la conception, l'écriture et l'édition des contenus et des supports de RP comme étant parmi les compétences les plus valorisées par les communicants interviewés. Ainsi, les compétences communicationnelle et journalistique définies, entre autres, comme rédactionnelles et écrites, occupent respectivement la première $(M=4.88)$ et la huitième place $(M=4.00)$. Cette étude souligne que la communication interne est parfois même confondue avec le seul travail d'écriture et d'édition de textes pour différentes publications internes, telles que les journaux internes par exemple (Ivanov, 2013 ; De Céglie et Ivanov, 2012). Ces compétences sont directement liées aux besoins en termes de circulation d'informations verticale et horizontale que chaque salarié éprouve (Clampitt et Downs, 2004) en tant que partie

\footnotetext{
${ }^{2}$ Le terme de RP internes est souvent utilisé comme synonyme de communication interne (Welch et Jackson, 2007), de relations internes (Grunig et Hunt, 1984) ou encore de relations avec les employés (Argenti, 1996). Nous considérons synonymes dans ce texte uniquement les RP internes et la communication interne, parce qu'elles couvrent les mêmes champs théoriques ainsi que des pratiques professionnelles semblables.
} 
prenante interne de l'organisation et font appel à la créativité et à l'imagination des relationnistes lors de la fabrique de contenus textuels. Les professionnels des RP jouent ainsi un rôle fondamental de production et de diffusion d'informations dans les organisations qui affectent directement la participation et l'engagement des publics internes, même si les supports de communication interne sont souvent suspectés de véhiculer les discours managériaux contrôlés en utilisant continuellement la langue de bois et la langue de coton (Morillon, 2005).

Au-delà de ces critiques formulées envers la communication interne depuis longtemps (Wild, 1953 ; Weil, 1990), l'écriture intelligible (Gregory, 2008) est une des valeurs ajoutées des RP dans les organisations, permettant non pas de séparer artificiellement «l'interne» et "l'externe» (ne parle-t-on habituellement pas de services de communication interne et externe ?), mais de les décloisonner. Le pivot central qui réunit ces deux pôles communicationnels est la compétence rédactionnelle : peu importe si le relationniste écrit pour les médias, les actionnaires ou les salariés de l'organisation, les compétences qui guident la pratique d'écriture restent toujours les mêmes.

Le Tableau 1 donne un aperçu des différentes études abordées portant sur les compétences des relationnistes tant en interne qu'en externe, en spécifiant les rôles que ces derniers jouent selon les auteurs cités.

\begin{tabular}{|c|c|c|}
\hline Auteurs - Travaux & Rôle & Compétences et tâches \\
\hline \multirow{4}{*}{$\begin{array}{l}\text { Broom, G. M. et Smith, G. D. } \\
\text { (1978). Toward an understanding } \\
\text { of public relations roles: An empir- } \\
\text { ical test of five role models' impact } \\
\text { on clients. Paper presented to the } \\
\text { Public Relations Division, Associa- } \\
\text { tion for Education in Journalism } \\
\text { annual convention, Seattle, WA. }\end{array}$} & L'expert & $\begin{array}{l}\text { Analyse de problèmes et } \\
\text { leurs solutions pratiques }\end{array}$ \\
\hline & Le technicien & $\begin{array}{l}\text { Réalisation d'outils et de } \\
\text { supports écrits (concevoir, } \\
\text { écrire, éditer...) }\end{array}$ \\
\hline & L'animateur & $\begin{array}{l}\text { Médiateur entre l'organi- } \\
\text { sation et ses différents pu- } \\
\text { blics }\end{array}$ \\
\hline & $\begin{array}{l}\text { Le facilitateur de } \\
\text { problèmes }\end{array}$ & $\begin{array}{l}\text { Prise de décision straté- } \\
\text { gique et recherche de solu- } \\
\text { tions sur des sujets de } \\
\text { haute importance }\end{array}$ \\
\hline $\begin{array}{l}\text { Piekos, J. et Einsiedel, E. (1990). } \\
\text { Roles and program evaluation tech- } \\
\text { niques among Canadian public re- } \\
\text { lation practitioners. In L. A. Grunig }\end{array}$ & Le manager & $\begin{array}{l}\text { Prise de décision straté- } \\
\text { gique ; capacités relation- } \\
\text { nelles et managériales }\end{array}$ \\
\hline
\end{tabular}




\begin{tabular}{|c|c|c|}
\hline $\begin{array}{l}\text { et J. E. Grunig (dir.), Public Rela- } \\
\text { tions Research Annual: Vol. } 2 \\
\text { (p. 95-113). New York, NY: } \\
\text { Routledge. }\end{array}$ & Le technicien & $\begin{array}{l}\text { Supporter le managemen } \\
\text { et réalisations techniques } \\
\text { (écrire, analyser, synthéti } \\
\text { ser, éditer...) }\end{array}$ \\
\hline
\end{tabular}
Routledge.

Le CEO ou le mana- Diriger, administrer et orger de haut niveau ganiser les RP

Beach, for Communicators. Communication World, 10(1), 12-15.

McGoon, C. (1995). 10 years from now. Communication World, 12(1), $18-22$.

Grunig, J. E. et Grunig, L. A. (2000). Public relation in strategic management of public relations: Theory and evidence from the IABC Excellence Project. Journalism Studies, 1(2), 303-321.
Le salarié non-cadre Écrire et éditer des supports de RP

Le relationniste intégré dans la coalition

Influencer la prise de décision stratégique
Cernicova, M., Dragomir, M. et Palea, A. (2011). Tentative conclusions regarding Romanian professional perceptions on the competences specific for PR specialists. Professional Communications and Translation Studies, 4(1-2), 3-10.
Le gestionnaire et le technicien

\begin{abstract}
Compétences professionnelles spécifiques et transversales : les compétences écrites et éditoriales sont représentées dans tous les domaines d'intervention du relationniste (interne et externe)
\end{abstract}

Le responsable ou le chargé de RP internes
Compétences communicationnelles (concevoir, parler, présenter...) et journalistiques (écrire, rédiger, éditer...)

Tableau $n^{\circ} 1$. Rôles et compétences des techniciens et des gestionnaires en RP.

Si un certain consensus sur l'importance de ces compétences existe, la difficulté de trouver une manière commune de les nommer est très révélatrice. Tantôt un savoirfaire communicationnel (McCleneghan, 2007 ; Wilcox et al., 2013), tantôt un savoirfaire informationnel (Berkowitz et Hristodoulakis, 1999), les habiletés écrites et éditoriales sont parfois même appelées des compétences journalistiques (Verčič, Verčič et Sriramesh, 2012). Cette confusion (ou amalgame ?) entre les compétences journalistiques et communicationnelles est extrêmement significative pour l'existence des RP, car elles ne relèvent ni complètement de la communication interne et externe, 
ni entièrement du journalisme, mais d'un entre-deux flou, qui trouve toute la raison de son existence dans des activités professionnelles en communication, exercées dans et pour les organisations, mais liées au savoir-faire journalistique.

\section{L’écriture : une compétence chère, mais rare des RP}

Malgré la réticence de conjuguer le savoir-faire journalistique avec les compétences techniques des relationnistes sur le plan paradigmatique (Mathien, 2001), la porosité entre les deux domaines professionnels a toujours été très présente. Il s'avère que l'un des fondateurs mêmes des RP dans le monde anglo-saxon, Ivy Lee, était un ancien journaliste professionnel qui a été un des premiers à mettre en place des supports écrits de communication, comme les newsletters, les bulletins internes et les communiqués de presse, à destination de publics internes et externes. Son travail, très apprécié et reconnu en son temps, était bâti sur une connaissance solide du métier journalistique, qu'il a su mettre au profit des organisations. De nombreux exemples contemporains d'anciens journalistes devenus communicants en Amérique du Nord et en Europe pourraient également être cités, surtout avec l'approfondissement de la crise du marché du travail journalistique et du financement des médias (cf. le dernier rapport «Étude sur les Médias et la publicité en ligne : transfert de valeur et nouvelles pratiques », commandé par le ministère français de la Culture et le Conseil supérieur de l'audiovisuel, publié en juillet $2018^{3}$ ). D'où l'intérêt et la pertinence de la problématique qui s'attache à traiter la source des compétences écrites et éditoriales en RP comme étroitement liée au journalisme. Autrement dit, au-delà de tout amalgame entre les métiers de journaliste et de communicant que nous désapprouvons ailleurs (Ivanov, 2014), il est pertinent de tenter d'expliquer comment un technicien de RP apprend à écrire et à éditer des supports lisibles si l'écriture n'est pas une compétence systématiquement et universellement issue des formations communicationnelles, mais journalistiques?

\subsection{L'enseignement : le chaînon manquant de la maîtrise des techniques écrites en RP ?}

Les formations en RP, au moins pour ce qui concerne le Canada et la France, ne proposent pas de programmes homogénéisés qui enseignent l'écriture en communication. Dans le cas du Canada, une des causes probables de ce constat est la typicité fédérale du pays, qui rend l'homogénéisation intra-universitaire impossible. Dans les

\footnotetext{
${ }^{3} \mathrm{http}$ //www.culture.gouv.fr/Thematiques/Audiovisuel/Rapports-etudes/Etude-sur-les-Medias-et-lapublicite-en-ligne-transfert-de-valeur-et-nouvelles-pratiques2
} 
universités francophones et anglophones canadiennes, la mise en place de programmes de premier et deuxième cycles labellisés en RP reste une initiative plutôt locale. Un effort pour fournir cette technicité aux étudiants de premier cycle au Canada est lié à la mise en place de programmes conjoints en RP entre les universités et les collèges (c'est le cas de l'Université d'Ottawa). L'idée est que ces derniers fournissent aux étudiants des compétences pratiques et que l'université leur donne la base théorique des RP. D'autres universités francophones, comme l'Université de Sherbrooke, l'Université de Montréal et l'Université du Québec à Montréal, proposent des programmes, des certificats et des cours de baccalauréat visant l'acquisition de compétences rédactionnelles en RP. Flynn et Sévigny (2009) soulignent que dans le monde anglo-saxon canadien, les programmes universitaires de RP de premier cycle accordent une certaine priorité aux formations communicationnelles plus générales, alors que les programmes spécialisés de Maîtrise en RP se font plutôt rares. Ils mettent en évidence le fait que les formations de deuxième cycle en RP n'ont été introduites que très tardivement (par exemple, en 2001 à McMaster University et en 2007 à DeGroote School of Business) et concluent que les formations théoriques et pratiques en RP devraient constituer, dans les années à venir, un des piliers des formations universitaires.

De l'autre côté de l'Atlantique, en France notamment, l'enseignement au niveau universitaire des techniques d'écriture et éditoriales en RP n'est que très peu présent dans les programmes de premier et de deuxième cycle. Une revue exhaustive des formations universitaires (Collet, Ivanov et Maas, 2016), réalisée sous l'égide de la Société Française des Sciences de l'Information et de la Communication (SFSIC), montre la quasi-absence de formations homologuées RP. Cette situation est sans doute liée au fait qu'en France (contrairement à la Belgique, l'Allemagne, l'Angleterre, ou encore la Slovénie, la Roumanie et la Bulgarie) l'appellation RP n'est pas du tout utilisée (on parle plutôt de communication stratégique, communication marketing ou encore de communication publique) au même titre qu'en Amérique du Nord (d'Almeida et Carayol, 2014). Par ailleurs, pour certains auteurs (Carayol, Chaudet et Frame, 2014), "parler de relations publiques, en France, a un côté suranné et presque vieillot » (p. 186), parce que ce terme fait référence aux pratiques normatives dépassées depuis les années 80 .

L'enseignement des techniques d'écriture et des compétences éditoriales en France dépend donc en grande partie de la bonne volonté des Départements de communication et des directeurs de programmes spécialisés (comme les Maîtrises et les Licences Professionnelles) qui ont une latitude relativement large pour inviter des enseignants (professionnels et chercheurs à temps partiel) spécialisés en RP. À titre d'exemple, c'est bien le cas de la Licence Professionnelle «Métiers de la communication : Chargé de communication» au sein de l'Université Paul-Valéry, Montpellier 3, qui se concentre sur la formation pratique des chargés des relations presse et relations médias. Il est à noter que le contenu pédagogique de ce type de programmes n'est ni homogénéisé ni généralisé. 
L'apprentissage des techniques journalistiques en RP se fait donc en grande partie sur le terrain (Kim et Johnson, 2011). Ehling (1992) démontre qu'il est très fréquent de pratiquer ce métier sans avoir obtenu un diplôme spécialisé dans le domaine des RP ou de communication dans un sens plus large. Pourtant, les disciplines connexes des RP, comme la sociologie, la psychologie et la gestion, ne se préoccupent pas fondamentalement des techniques d'écriture en entreprise. «La grande enquête : vers une meilleure connaissance des pratiques en communication dans les organisations canadiennes", pilotée par Marc David et Bernard Motulsky (2010), révèle que plus de la moitié des cadres de communication interviewés au Québec ont obtenu leur dernier diplôme via des formations autres qu'en communication (Administration des affaires, Arts et lettres, Sciences humaines, Droit et criminologie, etc.). C'est pourquoi, au Canada, des associations professionnelles comme la Société Canadienne de Relations Publiques (SCRP) sensibilisent la communauté des RP sur la nécessité d'assurer la formation et l'amélioration continue des compétences techniques des relationnistes, ce qui est actuellement au cour de ses préoccupations ${ }^{4}$.

La nécessité d'améliorer (ou de s'approprier) les techniques d'écriture et d'édition se fait donc toujours sentir, tant du côté des relationnistes que de celui des publics internes et externes. Une étude menée auprès des relationnistes membres de Public Relations Society of America (PRSA) et d'International Association of Business Communicators (IABC) par Kim et Johnson (2011) démontre qu'une large majorité de relationnistes considère la maîtrise des techniques d'écriture journalistique comme essentielle pour leur travail. Ils jugent que pour pratiquer efficacement le métier de relationniste, une spécialisation en journalisme est absolument nécessaire afin de mieux apprendre à écrire dans des styles et des formats variés. Or, pour Kim et Johnson (2011), ces compétences ne peuvent pas être acquises uniquement sur le terrain ou exclusivement par la voie de formations continues, car elles sont une combinaison complexe de formations universitaires spécialisées, de pratiques professionnelles quotidiennes et de savoir-faire personnel.

Le même sentiment est partagé du côté des destinataires privilégiés des professionnels des RP. Les journalistes, qui travaillent fréquemment avec les relationnistes par le biais des relations médias, ont une image désastreuse des professionnels des RP (Wilson et Supa, 2013 ; DeLorme et Fedler, 2003). Dans son étude internationale sur la convergence du journalisme et des RP, Macnamara (2015) souligne que les relationnistes ont une très « mauvaise presse » aux yeux des journalistes, parce que ces derniers travaillent habituellement avec des relationnistes inexpérimentés et peu habitués au travail journalistique et aux techniques d'écriture et d'édition. «Ils

\footnotetext{
${ }^{4} \mathrm{Cf}$. La discussion The Elevation of Public Relations: A discussion paper on a profession's present - and its possible future, (https://www.cprs.ca/getattachment/About/Who-We-Are/The-Future-ofCPRS/CPRS-Elevation_of_Public_Relations.pdf.aspx?lang=en-CA).
} 
pensent qu'envoyer un communiqué de presse suffit pour qu'il soit automatiquement repris par les journalistes » (Dr. Norman Swan, cité par Macnamara, 2015, p. 16), quel que soit le contenu ou la manière dont ce dernier a été écrit et construit. Sans vouloir réduire la méfiance des journalistes envers les relationnistes uniquement au manque de compétences écrites (bien d'autres facteurs peuvent être explicités), dans la pratique les communiqués de presse relookés et les rapports d'activités luxueux se transforment rapidement, aux yeux des journalistes, en « feuilles de chou» inutiles.

Il en est de même dans la pratique de la communication interne lorsque les relationnistes rédigent des contenus informationnels et les publient sur les Intranets et les journaux internes, en considérant que tous les membres du personnel vont lire et s'approprier les textes. Aux côtés des critiques traditionnelles de serviabilité formulées envers les RP internes, un des facteurs les plus fréquents du manque de réussite de la presse interne d'entreprise est son illisibilité (Ivanov, 2013).

\subsection{Faire rentrer le « journalisme » dans les organisations ?}

... Et si les tâches techniques dans les services de RP étaient réalisées par des (anciens) journalistes professionnels devenus relationnistes ? Au fond, cette idée peut choquer, mais elle n'est ni nouvelle ni inédite (Broustau et Francœur 2017). Elle a toujours été la source d'un pan de controverses et de débats, mais il nous semble qu'au $\mathrm{vu}$ des évolutions contemporaines des métiers des fonctions information et communication, ce débat mérite d'être relancé. Si en son temps Ivy Lee était un des premiers à emprunter la voie d'importation des compétences journalistiques vers les RP, aujourd'hui, l'influence des technologies socionumériques (Smith, 2008), la crise du journalisme et les besoins en termes de compétences écrites et éditoriales des organisations donnent un nouveau sens à cette idée.

L'embauche d'(anciens) journalistes par les organisations privées et publiques a fait naître une des formes les plus tangibles de cette hybridation, le journalisme d'entreprise, qui porte des missions rédactionnelles en externe comme en interne qui sont au service de l'entreprise (Ivanov, 2016a) : des missions qui s'inscrivent officiellement dans la stratégie de l'entreprise pour laquelle il travaille. En pratique, il est chargé de produire des contenus écrits, sonores, visuels, numériques, sur différents supports (papier, mais aussi électronique comme le Web 2.0, les Intranets, les blogs, les plateformes de discussions, d'échanges d'informations et de contenus, les réseaux sociaux d'entreprise). Pour ce faire, il élabore des articles de presse (reportage, interview, portrait, compte rendu, brève...), mais il peut également être amené à proposer des produits audio et vidéo. Ce travail inclut la recherche de sujets, de documentation et d'informations diverses qu'il collecte, catégorise, traite, synthétise, hiérarchise et rédige, d'une part en fonction de la stratégie de l'entreprise, d'autre part en suivant les techniques d'écriture journalistique (loi de proximité, angle, message, chute, attaque, titraille...) (Agnès, 2002). C'est bien le respect des procédés classiques 
de recherche, de collecte et de rédaction des informations collectées qui distinguent clairement son travail de celui d'un producteur de contenus, une activité floue qui s'est surtout développée avec la multiplication des plateformes et des réseaux socionumériques (cf. le débat lancé sur le site de la Fédération professionnelle des journalistes du Québec ${ }^{5}$ ).

Même si ce métier est bien identifié, défini et codifié par les organismes de recherche d'emploi, l'appellation «journaliste d'entreprise» est loin d'être universelle (on la rencontre beaucoup moins au Canada qu'en France et en Belgique, par exemple) et ne fait pas l'unanimité. Pourtant, ce métier se fait un chemin dans les organisations et c'est bien la pratique professionnelle du journalisme d'entreprise qui oblige la communauté scientifique à revoir sa légitimité. Il existe depuis des années des référentiels officiels (comme celui de l'Association pour l'emploi des cadres en France ${ }^{6}$ ) et professionnels ${ }^{7}$ des métiers de la fonction communication dans lesquels le journalisme d'entreprise est clairement décrit et identifié. D'autre part, certains organismes de formation et de conseil définissent explicitement ce métier ${ }^{8}$ afin de faciliter l'accès des futurs professionnels à ce marché. Ainsi, le métier de journaliste d'entreprise est défini sous le code ROME E1106 (dans la catégorie « Journalisme et information média $»)^{9}$ sur le site Internet de Pôle-Emploi en France.

En France, un effort remarquable a été fourni par l'association Communication et Entreprise, anciennement appelée Union des journaux et journalistes d'entreprise de France (UJJEF créée le 25 mars 1947 à Paris), en vue d'institutionnaliser ce métier depuis les années 1990. Une enquête, intitulée «Pour un statut de journaliste de presse d'entreprise», menée en 2002 pour l'UJJEF par le cabinet Occurrence, souligne dans son préambule : «Ni tout à fait journaliste sur le plan social et fiscal, ni tout à fait communicant par ses activités, le journaliste de la presse d'entreprise mérite que son métier soit apprécié à sa juste valeur et sa profession mieux reconnue des autorités. C'est dans ce sens que la Commission juridique engage la rédaction d'un livre blanc qui doit constituer l'un des éléments de base de cette action ». Les résultats de cette enquête stipulent en outre que la majorité des journalistes d'entreprise n'ont pas fait d'études journalistiques (seulement 14,5\% d'entre eux), mais de communication ou de sciences humaines et sociales. Ils allouent de 50 à $80 \%$

\footnotetext{
${ }_{6}^{5} \mathrm{https}: / /$ www.fpjq.org/producteur-de-contenu-mal-aime-journalisme/

${ }^{6} \mathrm{https} / / /$ recruteurs.apec.fr/Recrutement/Observatoire-de-1-emploi/Les-etudes-Apec-par-

thematique/Metiers-et-competences/Referentiel-des-metiers-cadres-de-la-fonction-communication

${ }^{7} \mathrm{http}: / /$ metiersdelacommunication.fr/journaliste-dentreprise-2/

${ }^{8} \mathrm{https} / / / \mathrm{www}$. iicp.fr/entreprise/metiers/journaliste-entreprise ;

http://www.studyrama.com/formations/fiches-metiers/journalisme/journaliste-d-entreprise-1102 ; http://www.letudiant.fr/metiers/secteur/communication/journaliste-d-entreprise_1.html ; http://www.cidj.com/article-metier/journaliste-d-entreprise ; https://www.kelformation.com/fichesmetiers/journaliste-d-entreprise.php

${ }^{9} \mathrm{http}: / / \mathrm{www}$.pole-emploi.fr/candidat/les-fiches-metiers-@/index.jspz?id=681
} 
de leur temps à la presse interne et exercent majoritairement une activité professionnelle de type « rédacteur ». Plus d'un tiers ne possèdent aucune carte de presse et deux tiers d'entre eux souhaitent obtenir le statut de «journaliste d'entreprise» et posséder une carte de presse.

Cet effort d'institutionnalisation du métier de journaliste d'entreprise en France n'est, certes, pas universel, mais la pratique de ce métier sous appellations différentes (journalisme de communication, par exemple) est loin d'être un phénomène isolé.

\subsection{Quelle place et quelle légitimité pour ce métier atypique?}

Travailler aux côtés d'anciens journalistes devenus communicants ne saurait-il permettre aux relationnistes de mieux développer leurs propres compétences rédactionnelles dans la mesure où la pratique quotidienne facilite leur acquisition continue ? Sans adopter une posture partisane, nous voyons deux contributions majeures à l'idée de faire entrer des journalistes d'entreprise dans les directions des RP : 1) contribuer à la formation et à l'amélioration des compétences rédactionnelles et éditoriales des relationnistes et 2) produire des supports d'information et de communication lisibles et écrits selon les règles de l'art. Pour que ces possibilités soient envisageables, embaucher simplement des anciens journalistes professionnels dans une direction de RP ne suffirait pas. Non pas parce que leurs compétences vont s'estomper ou disparaître lorsqu'ils quittent les rédactions journalistiques, mais parce que les structures organisationnelles qui les accueillent et les conditions d'attribution de leurs tâches professionnelles doivent être repensées et adaptées.

Constituer la légitimité de ce métier contesté et contestable nécessite, tout d'abord, la suppression de la séparation artificielle entre la communication interne et externe (Cheney et Christensen, 2001) et, en l'occurrence, entre les services et les tâches allouées aux relationnistes. Sur le terrain, une des tensions majeures qui accompagnent l'intégration des journalistes d'entreprise dans les organisations est la disparité de leur rattachement hiérarchique (Ivanov, 2016b). Lorsqu'ils sont embauchés uniquement pour s'occuper de la communication interne, ils se concentrent plutôt sur la production de publications internes écrites. S'ils sont plutôt engagés pour prendre en charge les relations presse, ils rédigent différents documents (comme les communiqués de presse et les contenus de site web) à destination des médias et des publics spécifiques (clients, utilisateurs de services, partenaires, collaborateurs, etc.). Or, écrire pour des publics hétérogènes - tant les salariés que les parties prenantes externes (Leitch et Neilson, 2001) - demande les mêmes compétences techniques et créatives. Un journal interne bien rédigé aura un succès semblable auprès des salariés qu'un communiqué de presse auprès des journalistes. Il convient donc de définir le paramètre d'intervention du journaliste d'entreprise et ses tâches quotidiennes non pas en se basant sur la séparation interne/externe largement répandue dans les RP 
(d'Almeida et Libaert, 2010), mais - bien au contraire - sur leur unification et harmonisation autour d'un axe professionnel lié à la production et à l'édition de supports informationnels et communicationnels. Le périmètre d'intervention du journaliste d'entreprise pourrait être élargi d'une manière transversale à d'autres domaines de l'organisation traditionnellement secondés par les RP.

Par ailleurs, en intégrant les organisations, la latitude rédactionnelle du journaliste d'entreprise fait face à un certain nombre d'écueils liés non seulement aux objectifs stratégiques de son employeur (du secteur public ou privé), mais aussi au fonctionnement des cercles managériaux. Pour mener à bien ses fonctions, l'emprise hiérarchique sur les corrections et les validations des écrits doit être élaguée. Les filtres managériaux (Both, 2006) via lesquels passent toutes les publications d'entreprise sont une réalité du fait que le journaliste d'entreprise travaille au sein d'une organisation - et d'une ou plusieurs directions - qui a une vision et des objectifs stratégiques définis. Il serait utopique de penser que tout contrôle hiérarchique sur les supports écrits et sur le processus rédactionnel pourrait être supprimé (ceci est loin d'être nécessaire !), mais pour mettre l'entreprise sous presse, le journaliste d'entreprise doit bénéficier d'une confiance accrue quant à la politique rédactionnelle et la réalisation de supports écrits. La langue de coton managériale (Morillon et Bouzon, 2009) va à l'encontre de la lisibilité des journaux internes et des communiqués de presse, même si au fond elle articule les visées stratégiques de l'organisation. Par ailleurs, il revient au journaliste d'entreprise de sensibiliser les instances hiérarchiques au danger de reprendre et de modifier le fond et la structure des textes écrits non pas pour mieux dire les choses, mais pour les « garnir ».

\begin{tabular}{|c|c|c|c|c|c|c|}
\hline $\begin{array}{l}\text { Formation } \\
\text { et carrière }\end{array}$ & $\begin{array}{l}\text { Rattache- } \\
\text { ment } \\
\text { hiérar- } \\
\text { chique }\end{array}$ & $\begin{array}{l}\text { Spectre } \\
\text { des tâches } \\
\text { allouées }\end{array}$ & $\begin{array}{l}\text { Spectre } \\
\text { d'inter- } \\
\text { vention }\end{array}$ & $\begin{array}{l}\text { Valida- } \\
\text { tions } \\
\text { hiérar- } \\
\text { chiques }\end{array}$ & $\begin{array}{l}\text { Fonction- } \\
\text { nement }\end{array}$ & $\begin{array}{l}\text { Régulation } \\
\text { interne }\end{array}$ \\
\hline $\begin{array}{l}\text { Université } \\
\text { ou école de } \\
\text { journa- } \\
\text { lisme. } \\
\text { Ancien } \\
\text { journaliste } \\
\text { profession- } \\
\text { nel }\end{array}$ & $\begin{array}{l}\text { Au direc- } \\
\text { teur des RP } \\
\text { ou à un } \\
\text { membre du } \\
\text { comité de } \\
\text { direction }\end{array}$ & $\begin{array}{l}\text { Communi- } \\
\text { cation } \\
\text { interne et } \\
\text { externe. } \\
\text { Activités } \\
\text { axées sur } \\
\text { l'écriture et } \\
\text { l'édition }\end{array}$ & $\begin{array}{l}\text { Transver- } \\
\text { sal. Les } \\
\text { sous-unités } \\
\text { organisa- } \\
\text { tionnelles } \\
\text { secondées } \\
\text { par la } \\
\text { direction } \\
\text { des RP }\end{array}$ & $\begin{array}{l}\text { Réduction } \\
\text { des filtres } \\
\text { managé- } \\
\text { riaux et } \\
\text { régulation } \\
\text { du proces- } \\
\text { sus de vali- } \\
\text { dation. } \\
\text { Confiance } \\
\text { et latitude } \\
\text { rédaction- } \\
\text { nelle }\end{array}$ & $\begin{array}{l}\text { Comité de } \\
\text { rédaction } \\
\text { constitué } \\
\text { par des re- } \\
\text { lationnistes } \\
\text { et salariés } \\
\text { des autres } \\
\text { secteurs } \\
\text { (proposi- } \\
\text { tions, sug- } \\
\text { gestions et } \\
\text { rédaction } \\
\text { de textes) }\end{array}$ & $\begin{array}{l}\text { Charte } \\
\text { rédaction- } \\
\text { nelle } \\
\text { approuvée } \\
\text { par la di- } \\
\text { rection }\end{array}$ \\
\hline
\end{tabular}

Tableau $n^{\circ} 2$. Prédispositions pour réguler les activités du journaliste d'entreprise et des métiers apparentés. 
In fine, il nous semble que constituer un comité de rédaction (Agnès et Durier, 1992), qui réunit des relationnistes œuvrant sur des tâches différentes, pourrait considérablement améliorer l'apprentissage par les professionnels des RP des techniques d'écriture et d'édition de supports écrits. Pour ce qui concerne la communication interne et la mise en place de supports internes, ce comité est devenu une norme (Ivanov, 2013, 2016b ; Détrie et Broyez, 2003). Les membres du comité y contribuent avec des idées et des suggestions de publications, mais ils peuvent aussi rédiger des textes sous la supervision du journaliste d'entreprise (actuellement, les corrections sont plutôt faites par un responsable des RP ou par un dircom. En étant secondé par un comité de rédaction, le journaliste d'entreprise peut, à la fois, intégrer les points de vue des différents relationnistes ou des autres salariés connaissant mieux leur domaine d'expertise, mais aussi les accompagner dans l'appropriation des techniques d'écriture et d'édition. Pour assurer le bon fonctionnement du comité, le journaliste d'entreprise peut œuvrer pour une charte professionnelle (Décaudin et Igalens, 2006) qui réglemente les différents échéanciers, procédures et règles. Cette charte permet de réguler la latitude rédactionnelle, les domaines d'intervention et le processus de corrections et de validations des publications du journaliste d'entreprise, ainsi que de limiter le paramètre d'intervention hiérarchique dans les différentes étapes de production de supports écrits.

\section{Conclusion}

Ouvrir les portes des organisations aux anciens journalistes n'est pas uniquement une question de compétences écrites et éditoriales. Cette hybridation - ou complémentarité - ne saurait exister sans prendre en compte plusieurs prédispositions nécessaires. Probablement celle qui est la plus compliquée à résoudre est l'impossibilité de faire converger la pratique du journalisme et des RP au niveau paradigmatique (Charron et De Bonville, 1997 ; Mathien, 2001) et, surtout, dans un contexte international (Macnamara 2015). En réalité, ces deux métiers ont des fondements culturels et historiques très différents des deux côtés de l'Atlantique. Si, dans la tradition nordaméricaine, l'émergence des études de la communication est plutôt liée aux sciences de gestion, dans la tradition européenne, elle est davantage ancrée dans les sciences humaines et sociales (Taylor et Delcambre, 2011). Il en est de même avec le journalisme dont l'origine et les pratiques connaissent des écarts considérables (cf. $L a$ recherche sur le journalisme : apports et perspectives ${ }^{10}$ ). Pour certains chercheurs, ces deux métiers initialement liés à des paradigmes différents ne sont pas détachables (Bougnoux, 2001), pour d'autres, il est important de les distinguer parce qu'ils articulent des pratiques et des techniques différentes qui ne doivent pas être

\footnotetext{
${ }^{10} \mathrm{https}$ ://lesenjeux.univ-grenoble-alpes.fr/2005/Seminaire_Gresec/index.php
} 
confondues (Miège, 2004). Ces débats démontrent qu'ils sont aujourd'hui encore la source de nombreuses discussions et controverses (Broustau et Francœur 2017), surtout dans le contexte du développement des médias socionumériques qui ont accéléré la convergence entre le journalisme et les RP. Alors, un métier hybride peutil exister sans une reconnaissance et un consentement paradigmatique ?

Oui ! Il existe déjà dans les organisations depuis plusieurs années. Il est également reconnu par plusieurs associations professionnelles et par certains organismes étatiques de recherche d'emploi. Il est aussi bien reconnu du côté des organisations, qui embauchent sciemment d'anciens journalistes professionnels afin d'améliorer leur presse d'entreprise et leurs relations médias. Mais cette reconnaissance est-elle partagée sur le terrain par les acteurs concernés par la pratique du journalisme d'entreprise : les journalistes professionnels, les relationnistes et les journalistes d'entreprise ? Si les tâches professionnelles des relationnistes et des journalistes constituent les deux côtés de la même monnaie (Evans, 2010), le passage vers l'autre côté des barricades pose un double problème. Tout d'abord, l'impossibilité de s'auto-identifier comme journaliste d'entreprise (c'est bien le cas au Canada où aucun relationniste ne s'auto-identifie comme journaliste d'entreprise dans «La grande enquête : vers une meilleure connaissance des pratiques en communication dans les organisations canadiennes »). Ensuite, il y a la difficulté d'être accepté par ses anciens confrères comme un journaliste œuvrant en entreprise, ce qui reviendrait pour les journalistes professionnels à remettre en question le fondement même de leur métier (Agnès, 2002). Le problème de l'identification à la pratique du journalisme d'entreprise se pose tant du côté des publics externes que des parties prenantes internes. Une activité professionnelle à laquelle il est difficile de s'(auto)identifier, pourrait-elle constituer les bases d'un métier hybride dans les organisations?

Non, si nous ne prenons pas en compte la dimension éthique de l'hybridation entre le journalisme et les RP. De la complexité à définir l'éthique (Bernier, 2004) à la difficulté de concevoir la convergence entre l'éthique du journaliste et celle du relationniste : l'épineuse question du croisement impossible entre le journalisme et les RP est toujours d'actualité (Macnamara, 2015). Pour certains auteurs (Ladendorf, 2012), les frontières entre les pratiques journalistiques et les RP se sont considérablement estompées. Il existerait ainsi dans les codes éthiques des zones de convergences, notamment lorsqu'il s'agit de la codification de la conduite individuelle et des critères de professionnalisme, des standards moraux et de l'expertise spécifique (Yang, Taylor et Saffer, 2016). Mais au niveau pratique, une question se pose: le journaliste d'entreprise est-il capable de préserver son éthique personnelle et professionnelle dans les organisations telle que conçue par l'art journalistique ? Ou doit-il plutôt adopter l'éthique défendue et réclamée par le professionnel des RP (Grunig, 2014), qui a certes des zones de convergences avec celle du journalisme, mais qui se distingue considérablement d'elle? Même si l'information est considérée comme le contenu cognitif de la communication (Miège, 2004), qui fournit du sens aux productions textuelles, le 
découpage classique entre l'information et la communication s'opère par des tensions entre les pratiques journalistique et communicationnelle (Martin-Lagardette, 2006). D'où la difficulté d'inclure le journalisme d'entreprise ou les métiers apparentés dans les codes éthiques en RP, dont le manque laisse pour l'instant le travail des relationnistes en autorégulation (Holtzhausen, 2015).

Au-delà des difficultés, des débats et des controverses soulevés ici, le technicien chargé d'écrire et d'éditer des supports d'information et de communication a la rude tâche de sensibiliser et de convaincre le management aux enjeux liés au cœur de son activité : écrire dans et pour les organisations. Et ce processus commence probablement avec la volonté et la capacité de l'autodétermination et de l'auto-identification comme professionnel (peu importe le nom du métier) qui porte les enjeux des compétences de l'écriture journalistique au profit des organisations et de leurs publics internes et externes.

\section{Bibliographie}

Agnès, Y. (2002). Manuel de journalisme : écrire pour le journal. Paris, France : La Découverte.

Agnès, Y. et Durier, M. (1992). L'entreprise sous presse. Paris, France : Dunod.

Argenti, P. (1996). Corporate communication as a discipline: toward a definition. Management Communication Quarterly, 10(1), 73-97.

Berkowitz, D. et Hristodoulakis, I. (1999). Practitioner Roles, Public relations Education, and professional Socialization: An Exploratory Study. Journal of Public Relations Research, 11(1), 91-103.

Bernier, M.-F. (2004). Éthique et déontologie du journalisme. Sainte-Foy, Québec : Les Presses de l'Université Laval.

Both, A. (2006). Le journal interne d'entreprise. Ethnographie d'une mission impossible? Ethnologie française, 36(1), 45-54.

Bougnoux, D. (2001). Introduction aux sciences de la communication (coll. Repère). Paris, France : La Découverte.

Bowen, A. S., Rawlins, B. et Martin, T. (2010). An Overview of the Public Relations Function. New York, États-Unis : Business Expert Press, LCC.

Bronn, P. S. (2001). Communication managers as strategists? Can they make the grade? Journal of Communication Management, 5(4), 313-326.

Broom, G. M. (1982). A comparison of sex roles in public relations. Public Relations Review, 8(3), 17-22. 
Broom, G. M. et Dozier, D. M. (1986). Advancement for Public Relations Role Models. Public Relations Review, 12(1), 37-56.

Broom, G. M. et Smith, G. D. (1978). Toward an understanding of public relations roles: An empirical test of five role models' impact on clients. Paper presented to the Public Relations Division, Association for Education in Journalism annual convention, Seattle, WA, États-Unis.

Broom, G. M. et Smith, G. D. (1979). Testing the practitioner's impact on clients. Public Relations Review, 5(3), 47-59.

Broustau, N. et Francœur, C. (2017). Relations publiques et journalisme à l'ère numérique. Québec : Presses de l’Université du Québec.

Carayol, V., Chaudet, B. et Frame, A. (2014). Quelques jalons pour une histoire des relations publiques en France, des années 1900 jusqu'au début des années 80 . Recherches en communication, 41, 185-202.

Cernicova, M., Dragomir, M. et Palea, A. (2011). Tentative conclusions regarding Romanian professional perceptions on the competences specific for PR specialists. Professional Communications and Translation Studies, 4(1-2), 3-10.

Charron, J. et De Bonville, J. (1997). Le paradigme du journalisme de communication. Communication, 17(2), 50-97.

Cheney, G. et Christensen, L. T. (2001). Public Relations as Contested Terrain: A critical Response. Dans R. L. Heath et G. Vasquez (dir.), Handbook of Public Relations (p. 167-182). Thousand Oaks, CA, États-Unis : Sage.

Clampitt, P. G. et Downs, C. W. (2004). Downs-Hazen Communication Satisfaction Questionnaire. Dans C. W. Downs et A. D. Adrian (dir.), Assessing organizational communication (p. 139-157). London, Royaume-Uni : Guilford.

Collet, L., Ivanov, I. et Maas, E. (2016). Cartographie des formations en SIC par la Société Française des Sciences de l'Information et de la Communication (SFSIC) : usages des données et méthodes de visualisation. Communication et Organisation, 49, 241-250.

D’Almeida, N. et Carayol, V. (2014). La communication organisationnelle, une question de communauté. Revue Française des Sciences de l'information et de la communication, 4, mis en ligne le 01 janvier 2014, consulté le 20 août 2018. URL : http://journals.openedition.org/ rfsic/870 ; DOI : 10.4000/rfsic.870.

D'Almeida, N. et Libaert, T. (2010). La communication interne de l'entreprise ( 6 éd.). Paris, France : Dunod. 
David, M. D. et Motulsky, B. (2010). La grande enquête: vers une meilleure connaissance des pratiques en communication dans les organisations canadiennes.

http://www.crp.uqam.ca/pages/docs/centres/Resultats_GE_2011.pdf

Décaudin, J.-M. et Igalens, J. (2006). La communication interne. Paris, France : Dunod.

De Ceglie, A. et Ivanov, I. (2012). Le journal interne : une pratique collaborative de connaissance. Dans W. Mustafa El Hadi (dir.), Organisation des connaissances : dynamique et stabilité (p. 183-194), Hermès Science, 11(416).

De La Broise, P. et Brulois, V. (2010). La communication interne aux prises avec la professionnalisation. Communiquer, 3(4), 123-134.

De Lorme, D. et Fedler, F. (2003). Journalists' hostility toward public relations: An historical analysis. Public Relations Review, 29, 99-124.

Détrie, P. et Broyez, C. (2003). La communication interne au service du management. Paris, France : Éditions Liaisons.

Dozier, D. M. (1981). The diffusion of evaluation methods among public relations practitioners. Paper presented at the annual convention of the Association for Education in Journalism, Public Relations Division. East Lansing, MI, ÉtatsUnis.

Dozier, D. M. (1992). The organizational roles of communications and public relations practitioners. Dans. J. E. Grunig (dir.), Excellence in public relations and communication management, (p. 327-356). Hillside, NJ, États-Unis : Lawrence Erlbaum Associate, Inc.

Edwards, L. (2011). Defining the "object" of public relations research: A new starting point. Public Relations Inquiry, 1(1), 7-30.

Ehling, W. P. (1992). Public relations education and professionalism. Dans J. E. Grunig (dir.), Excellence in public relations and communication management (p. 439-464). Hillside, NJ, États-Unis : Lawrence Erlbaum Associate, Inc.

Evans, T. (2010). We are all in PR now. British Journalism Review, 21(2), 31-36.

Flynn, T. (2014). Do They Have What It Takes? A review of the Literature on Knowledge, Competencies, and Skills Necessary for Twenty-First-Century Public Relations Practitioners in Canada. Canadian Journal of Communication, 39(3), 361-384.

Flynn, T. et Sévigny, A. (2009). The Paradox of Public relations/Communications management Education in Canada: Taught But Not Studied. The McMaster 
Journal of Communication. 6(1). Article 2. URL : https://journals.mcmaster.ca/ $\mathrm{mjc} /$ article/view/244

Fondin, H. (2001). La science de l'information: posture épistémologique et spécificité disciplinaire. Documentaliste-Sciences de l'Information, 2(38), 112122.

Gregory, A. (2008). Competencies of senior communication practitioners in the UK. Public Relations Review, 34, 215-223.

Grunig, J. E. (1992). Excellence in public relations and communication management. Hillsdale, NJ, États-Unis : Lawrence Erlbaum Associates.

Grunig, J. E. (2006). Furnishing the Edifice: Ongoing Research on Public Relations as a Strategic Management Function. Journal of Public Relations Research, 18(2), 151-176.

Grunig, J. E. (2014). Ethics problems and theories in public relations. Revue de communication sociale et publique, 11, 1-14.

Grunig, J. E. et Grunig, L. A. (2000). Public relation in strategic management of public relations: Theory and evidence from the IABC Excellence Project. Journalism Studies, 1(2), 303-321.

Grunig, J. et Hunt, T. (1984). Managing Public Relations. New York, États-Unis : Holt, Rinehart and Winston.

Holtzhausen, D. R., (2015). The unethical consequences of professional communication codes of ethics: A postmodern analysis of ethical decisionmaking in communication practice. Public Relations Review, 41, 769-776.

Ivanov, I., (2013). Communiquer et agir en commun : le cas d'un journal interne instrumentalisé. Communication et Organisation, 43, 213-226.

Ivanov, I. (2014). Du journal grande presse au journal d'entreprise. Les (anciens) journalistes entre l'information et la communication. SEMEN, Revue sémiolinguistique des textes et des discours, 48, 135-148.

Ivanov, I. (2016a). Le journalisme d'entreprise, un métier méconnu et controversé. Mondes Sociaux. URL : http://sms.hypotheses.org/

Ivanov, I. (2016b). Que font les communicants pour sauver leur métier ? Étude de cas d'un service de communication en mal de reconnaissance. Communication et professionnalisation, 4, 78-99.

Jeffery, L. et Brunton, M. (2010). Identifying competencies for communication practice: A needs assessment for curriculum development and selection in New Zeeland. Public Relations Review, 36, 202-205. 
Kim, E. et Johnson, T. L. (2011). Making the Grade: What Constitutes a Successful PR Education? 14th International Public Relations Research Conference Pushing the Envelope in Public Relations Theory and Research and Advancing Practice, Miami, Florida, March 9-12th, 458-467.

Ladendorf, M. (2012). Freelance journalists' ethical boundary settings in information work. Nordicom Review, 33(1), 83-98.

Leitch, S. et Neilson, D. (2001). Bringing Publics Into Public Relations: New Theoretical Frameworks for Practice. Dans R. L. Heath et G. Vasquez, (dir.), Handbook of Public Relations (p. 127-138). Thousand Oaks, CA, États-Unis : Sage.

Macnamara, J. (2015). The Continuing Convergence of Journalism and PR: New Insights for Ethical Practice From a Three-Country Study of Senior Practitioners. Journalism \& Mass Communication Quarterly, 93(1), 1-24.

Maisonneuve, D. (2010). Les relations publiques dans une société en mouvance (4 éd.). Québec : Presses de l'Université du Québec.

Martin-Lagardette, J.-L. (2006). L’information responsable. Un défi démocratique. Paris, France : Éditions Diffusion Charles Léopold Mayer.

Mathien, M. (1998). Le journalisme professionnel face aux mutations de l'information et de la communication: déroute des valeurs ou réaction? Quaderni, « McLuhan, trente ans après », 37, 11-42.

Mathien, M. (2001). Le journalisme de communication : critique d'un paradigme spéculatif de la représentation du journalisme professionnel. Quaderni, « Figures du journalisme : critique d'un imaginaire professionnel », 45, 105-135.

McCleneghan, J. S. (2007). The PR counselor vs. PR executive: What skill sets divide them? Public Relations Quarterly, 52(4), 15-17.

McGoon, C. (1993). Life's a Beach, for Communicators. Communication World, $10(1), 12-15$.

McGoon, C. (1995). 10 years from now. Communication World, 10(1), 18-22.

Miège, B. (2004). L’information - communication, objet de connaissance. Bruxelles, Belgique : De Boeck.

Morillon, L. (2009). Le journal de communication interne, un agent des enjeux stratégiques organisationnels. Semen, 28, 31-51.

Morillon, L. et Bouzon, A. (2009). Les injonctions paradoxales de la communication interne en période de tensions organisationnelles. Études de communication, 33, 189-202. 
Niquette, M. (2000). De la féminisation des relations publiques: un discours en rupture de l'histoire des femmes relationnistes. Recherches féministes, 13(2), 4775 .

Piekos, J. et Einsiedel, E. (1990). Roles and program evaluation techniques among Canadian public relation practitioners. Dans L. A. Grunig et J. E. Grunig (dir.), Public Relations Research Annual: Vol. 2 (p. 95-113). New York, NY, ÉtatsUnis : Routledge.

Ross, L. (2005). L'écriture de presse : l'art d'informer (2 $2^{\mathrm{e}}$ éd.). Montréal, Canada : Chenelière Éducation.

Sallot, L. et Johnson, E. (2006). Investigating relationship between journalists and public relations practitioners. Work together to set, frame and build the public agenda, 1991-2004. Public Relations Review, 32, 151-159.

Smith, B. (2008). [Review of A complicated, antagonistic, symbiotic affair: Journalism, public relations and their struggle for public attention]. Journalism \& Mass Communication Quarterly, 85, 925-927.

Taylor, J. R. et Delcambre, P. (2011). La communication organisationnelle : histoire, enjeux et fondements. Dans S. Grosjean et L. Bonneville (dir.). Communication organisationnelle: Approches, processus, enjeux (p. 2-31). Montréal, Canada : Chenelière Éducation.

Verčič, A. T., Verčič, D. et Sriramesh, K. (2012). Internal communication: definition, parameters, and future. Public Relations Review, 38, 223-230.

Verčič, D. (2000). The European public relations body of knowledge. Journal of Communication Management, 4(4), 341-351.

Weil, P. (1990). Communication oblige! Communication institutionnelle et de management. Paris, France : Les Éditions d'Organisation.

Welch, M. et Jackson, P. (2007). Rethinking internal communication: a stakeholder approach. Corporate Communication: An International Journal, 12(2), 177-198.

Wilcox, D. L., Cameron, G. T., Reber, B. H. et Shin, J. (2013). Think public relations. Upper Saddle River, NJ, États-Unis : Pearson Education.

Wild, J. (1953). Employee Journal. Business Quarterly, 17(4), 225-239.

Wilson, D. et Supa, D. (2013). Examining modern media relations: An exploratory study of the effect of Twitter on the public-relations-journalist relationship. Public relations Journal, 7(3), 1-20. 
Yang, A., Taylor, M. et Saffer, A. J. (2016). Ethical convergence, divergence or communitas? An examination of public relations and journalism codes of ethics. Public Relations Review, 42(1), 146-160.

Yeomans, L. (2006). Internal communication. Dans R. Tench et L. Yeomans (dir.), Exploring Public relations (p. 332-352). Upper Saddle River, NJ, États-Unis : Pearson Education. 